\title{
Aflibercept in Diabetic Retinopathy
}

\author{
Abhinav R Bheemidi, ${ }^{1}$ Carolina CS Valentim² and Rishi P Singh ${ }^{2}$ \\ 1. Case Western Reserve University School of Medicine, Cleveland, OH, USA; 2. Center for Ophthalmic Bioinformatics, Cole Eye Institute, \\ Cleveland Clinic, Cleveland, OH, USA
}

DOI: https://doi.org/10.17925/OPHT.2021.15.1.18

W th the growing prevalence of diabetes mellitus in the USA, diabetic retinopathy (DR) cases are expected to increase. Panretinal photocoagulation (PRP) is considered standard of care for the treatment of DR, but it can be associated with vision impairment secondary to tissue destruction. Recently, anti-vascular endothelial growth factor (anti-VEGF) agents emerged as a promising alternative to PRP. They have been proven safe and superior to PRP in treating DR. The most recently developed anti-VEGF agent, aflibercept, which has been approved by the US Food and Drug Administration, is a recombinant fusion protein with antiangiogenic activity in multiple pathways. Several clinical trials, summarized here, highlight aflibercept's advantages when used in DR treatment.

\section{Keywords}

Anti-VEGF therapy, recombinant fusion protein, aflibercept, diabetic retinopathy, proliferative diabetic retinopathy

Disclosure: Rishi P Singh has received personal fees from Genentech/Roche, Alcon/Novartis, Zeiss, Bausch + Lomb and Regeneron Pharmaceuticals, Inc.; and grants from Apellis and Graybug. Abhinav R Bheemidi and Carolina CS Valentim have no financial or non-financial relationships or activities to declare in relation to this article.

Review process: Double-blind peer review.

Compliance with ethics: This study involves a review of the literature and did not involve any studies with human or animal subjects performed by any of the authors.

Authorship: The named authors meet the International Committee of Medical Journal Editors (ICMJE) criteria

for authorship of this manuscript, take responsibility

for the integrity of the work as a whole, and have

given final approval for the version to be published.

Access: This article is freely accessible at

touchOPHTHALMOLOGY.com (C) Touch Medical Media 2021

Received: 6 April 2021

Accepted: 3 June 2021

Published online: 12 July 2021

Citation: touchREVIEWS in Ophthalmology. 2021; 15(1):18-22

Corresponding author: Rishi P Singh, Cole Eye Institute, Cleveland Clinic, 9500 Euclid Ave, i-32, Cleveland, OH 44195, USA. E: singhr@ccf.org

Support: No funding was received in

the publication of this article.
The National Diabetes Statistics Report 2020 estimates that of the 34.1 million adults in the USA with diabetes in 2018, 21.4\% had undiagnosed disease. ' Due to an insidious disease course, diabetic complications like diabetic retinopathy (DR) are common. Among US diabetic adults, $28.5 \%$ have DR and $4.4 \%$ have vision-threatening DR. ${ }^{2}$ DR remains the leading cause of new cases of blindness in American adults, representing a major public health concern. ${ }^{3.4}$

DR development and progression are associated with diabetes duration, poor glycaemic control, systemic hypertension and microalbuminuria. ${ }^{5-7}$ Without proper management, DR progresses in an orderly fashion from nonproliferative DR (NPDR) to proliferative DR (PDR). Annual progression from mild NPDR to PDR ranged from 0\% to $3.3 \%$ in a systematic review. ${ }^{6}$ Regarding visual function, the vision-related functional burden of mild or moderate NPDR is comparable to diabetic patients without DR, while it is significantly higher among people with severe NPDR and PDR. ${ }^{8}$ PDR, which is characterized by neovascularization and has a global prevalence of $1.4 \%$, is associated with a significantly higher risk of vision loss than NPDR. ${ }^{9,10}$ Almost half of eyes with PDR will progress to severe vision loss (Figure 1). ${ }^{11}$

Inflammation and angiogenesis are the drivers of DR development and progression. Bloodretinal barrier breakdown and neovascularization occur secondary to relative retinal ischaemia, which promotes upregulation of vascular endothelial growth factor (VEGF, isoforms A-E), their receptors (VEGFR, isoforms 1-3), placental growth factor (PIGF) and others. ${ }^{12-14}$ Panretinal photocoagulation (PRP) has been the standard of treatment for PDR and reduces the risk of severe vision loss by over 50\%. ${ }^{11}$ PRP reduces the drive for angiogenesis, with a corresponding decrease in VEGF post-treatment. ${ }^{15}$ Since PRP involves the destruction of ischaemic retina, it is associated with permanent impairment of visual function, including restricted visual fields, loss of colour vision, reduced contrast sensitivity, impaired night vision and increased incidence of macular oedema. ${ }^{16-18}$ In recent years, molecules that directly target VEGF have been developed, and multiple trials showed they are viable alternatives to treat DR in various stages and adjuncts to PRP treatment. Bevacizumab, ranibizumab and aflibercept are anti-VEGF agents currently available for intravitreal use, although the first is off-label. All three agents were proved to be safe and efficacious for the treatment of diabetic macular oedema (DMO). ${ }^{19-21}$

Anti-VEGF therapy is not without limitations. Since the treatment is provided via repeated intravitreal injections, there is a requirement for consistent follow-up. The long-term effect of anti-VEGF treatment on retinal ischaemia remains to be elucidated. Upon cessation of therapy, it has been shown that oedema and neovascularization can reoccur.22 In younger patients, this uncertainty of long-term outcomes is a concern when determining treatment. Anti-VEGF treatment is also associated with a higher cost relative to PRP. For each line of vision saved, the estimated cost of anti-VEGF therapy $(\$ 16,489)$ was more than double that of PRP $(\$ 7,252)$. In the facility setting, the cost utility for PRP was $85 \%$ lower than anti-VEGF therapy. ${ }^{23}$

Aflibercept is the most recently approved anti-VEGF agent for intravitreal use. It is a recombinant fusion protein comprising the binding domains of VEGF-1 and VEGF-2 receptors fused with the FC 
Figure 1: Neovascularization of the iris (NVI) can develop in eyes with untreated proliferative diabetic retinopathy; NVI often leads to neovascular glaucoma, an important cause of blindness and enucleation

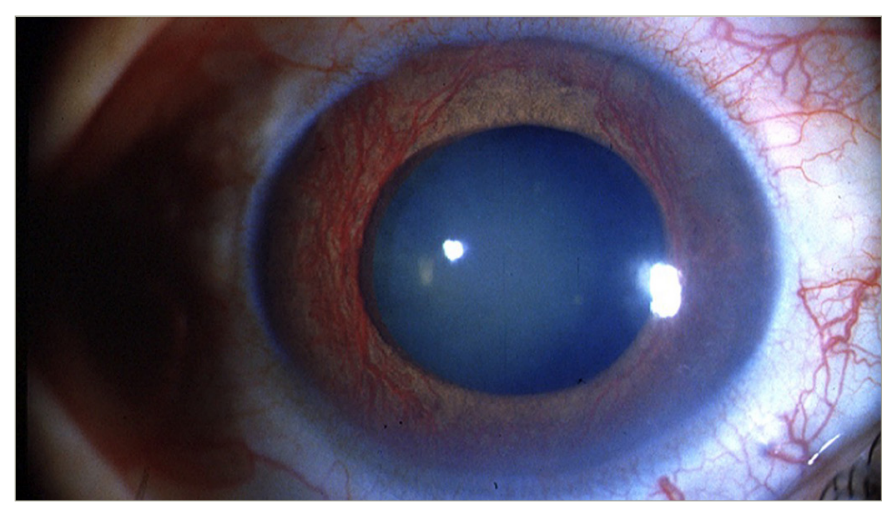

portion of human immunoglobulin G1. As a result, it binds to VEGF with high affinity with extended in vivo half-life. ${ }^{24}$ Aflibercept shows activity against all isoforms of VEGF-A, as well as VEGF-B, C, D and PIGF. ${ }^{25}$ VEGF-A binding is the principal mechanism of action; however, PIGF inhibition is critical. PIGF acts on endothelial cells by interacting with VEGFR-1 and increases availability of VEGF-A to bind VEGFR-2, thereby mediating leakage and angiogenesis. ${ }^{26}$ Furthermore, PIGF levels in DR eyes have been correlated with progressive retinal ischaemia and VEGF-A levels, and PIGF deletion/inhibition animal models have been associated with decreased leakage and inflammation. ${ }^{27}$ There has been significant exploration regarding the potential uses of aflibercept for both NPDR and PDR. The purpose of this article is to summarize findings from recent clinical trials (Table 1) and review the impact of these developments on clinical practice for the use of aflibercept.

\section{The PANORAMA study}

PANORAMA was a multicentre, double-masked, controlled, phase III clinical study, which examined the efficacy and safety of treatment with intravitreal aflibercept versus sham in patients with moderately severe to severe NPDR without centre-involved diabetic macular oedema $(\mathrm{Cl}-$ $\mathrm{DMO}){ }^{28,29}$ Four hundred and two patients were randomized to receive $2 \mathrm{mg}$ of aflibercept in one of two dose regimens or sham. The first aflibercept dose regimen was five monthly injections, followed by one injection every 8 weeks (2 mg every 8 weeks [2q8]) until week 52, and then pro re nata (PRN; as needed) dosing. The second aflibercept dose regimen was three monthly injections, followed by one injection after 8 weeks, and then one injection every 16 weeks (2q16).

In the first year, the proportion of patients with $\mathrm{a} \geq 2$-step improvement in diabetic retinopathy severity score (DRSS) was higher in the aflibercept groups $(2 q 8,2 q 16)$ versus sham ( $80 \%$ and $65 \%$, respectively, versus $15 \%$; $\mathrm{p}<0.0001) .28,29$ The proportion of patients who progressed to PDR or had evidence of neovascularization was lower in the aflibercept groups $(2 q 8,2 q 16)$ versus sham ( $3 \%$ and $4 \%$, respectively, versus $20 \%$; $p<0.001)$. These results were maintained in the second year. ${ }^{29}$ Of note, $92 \%$ of eyes that achieved $\mathrm{a} \geq 2$-step improvement in DRSS at year 1 maintained the improvement from baseline with decreased dosing through week 100. The second-year results speak to the impact of different dosing regimens. The proportion of the PRN patients (2q8) with a $\geq 2$-step improvement from baseline in DRSS decreased from $80 \%$ at 52 weeks to $50 \%$ at 100 weeks. In contrast, patients who continued with the 16-week injection schedule (2q16) displayed consistent $\geq 2$-step improvement in DRSS, with $65 \%$ at 52 weeks and 62\% at 100 weeks. PDR and/or anterior segment neovascularization occurred in 4\% of the PRN group, compared with $3 \%$ among those who received injections every 16 weeks, and $20 \%$ of the sham patients.

\section{The VIVID and VISTA studies}

VIVID and VISTA were phase III, double masked, active controlled, randomized, 148-week trials. A total of 862 patients with $\mathrm{Cl}$-DMO and best corrected visual acuity (BCVA) between 24 and 73 Early Treatment Diabetic Retinopathy Study (ETDRS) letters were included in the study. ${ }^{30,31}$ Approximately $5 \%$ of these patients had PDR at baseline. Patients were randomly assigned to receive $2 \mathrm{mg}$ of aflibercept every 4 weeks (2q4), $2 \mathrm{mg}$ of aflibercept every 8 weeks after five initial monthly doses (2q8), or macular photocoagulation at baseline with sham injections at each visit. Retrospective analyses of VIVID/VISTA examined the impact of intravitreal aflibercept on DR progression and the impact of baseline DRSS on visual outcomes. ${ }^{32,33}$

The week 52 and week 100 results indicate that the proportion of patients who showed a $\geq 2$-step improvement in DRSS was greater in the pooled aflibercept group compared with the photocoagulation group (week 52 : $31 \%$ versus $12 \%, p<0.0001$; week 100 : $35 \%$ versus $13 \%, p<0.0001) .{ }^{32}$ The week 52 results showed a significant difference in change from baseline BCVA in the $2 \mathrm{q} 4$ and $2 \mathrm{q} 8$ groups relative to the photocoagulation group of $12.5 \pm 9.5$ letters and $10.7 \pm 8.2$ letters compared with $0.2 \pm 12.5$ letters $(p<0.0001)$ in VISTA, and $10.5 \pm 9.5$ letters and $10.7 \pm 9.3$ letters compared with $1.2 \pm 10.6$ letters $(p<0.0001)$ in VIVID, respectively. ${ }^{30}$ The proportion of patients in whom PDR developed was smaller in the pooled aflibercept group compared with the photocoagulation group (week 52: $2 \%$ versus $7 \%, p=0.0002$; week 100 : $2 \%$ versus $9 \%, p<0.0001) .{ }^{32}$

Even after adjusting for baseline BCVA, the treatment effect between aflibercept and photocoagulation had an increasing trend as DRSS increased, with the highest treatment effect found in patients with DRSS $\geq 53 .{ }^{33}$ In addition, a greater proportion of patients with DRSS $\geq 53$ had a $\geq 2$-step improvement in DRSS compared with other patients. These results suggest a difference in treatment effect based on baseline DRSS, although the authors note the analysis to be underpowered to make this determination.

\section{The CLARITY study}

CLARITY was a prospective, single-blind, randomized, controlled, phase IIb, multicentre, non-inferiority trial examining the safety and efficacy of aflibercept versus PRP for BCVA in PDR patients, as measured by ETDRS letters. ${ }^{34}$ A total of 221 patients with no coexisting ocular disease and evidence of untreated PDR or PDR treated with PRP unsuccessfully were randomly assigned to the aflibercept group or PRP group. In the aflibercept group, $2 \mathrm{mg}$ of aflibercept was intravitreally injected at baseline, 4 weeks, and 8 weeks with follow-up visits every 4 weeks thereafter, during which, injections were given PRN. PRP was delivered at baseline and in fractionated fortnightly sessions with a follow-up at week 12 .

Aflibercept was both superior and non-inferior in BCVA compared with PRP at week 52 (mean difference in intent to treat population was 3.9 letters [2.3-5.6, $p<0.0001]$ and in per-protocol was 4.0 letters [2.4-5.7, $p<0.0001])$. With regard to loss of visual acuity, 5 of 107 patients receiving aflibercept had a $\geq 10$ letter worsening in BCVA compared with 16 of 104 patients in the PRP group (difference of 11\% [2.6-19.3, $p=0.009]$ ). With regard to anatomic outcomes at 52 weeks, $89 \%$ of patients in the aflibercept group had no macular oedema at 52 weeks compared to $71 \%$ of patients in the PRP group. Similarly, the occurrence of vitreous 
Table 1: Summary of clinical trials comparing aflibercept and other treatments for diabetic retinopathy

\begin{tabular}{|c|c|c|c|c|c|c|}
\hline Trial & $\begin{array}{l}\text { No. of } \\
\text { eyes }\end{array}$ & Diagnosis & Intervention & Follow-up & Outcome & Comments \\
\hline PANORAMA $^{28,29}$ & 402 & $\begin{array}{l}\text { Moderately severe } \\
\text { to severe NPDR } \\
\text { without CI-DMO }\end{array}$ & $\begin{array}{l}\text { IVI aflibercept } 2 \mathrm{mg} \\
2 \mathrm{q} 8 \text { versus } \\
\text { IVI aflibercept } 2 \mathrm{mg} \\
2 \mathrm{q} 16 \text { versus sham }\end{array}$ & 100 weeks & $\begin{array}{l}\text { Proportion of eyes with } \geq 2 \text {-step improvement in } \\
\text { DRSS at week } 52 \text { (80\% versus } 65 \% \text { versus 15\%) } \\
\text { Proportion of eyes with PDR or ASNV at week } 52 \\
\text { (3\% versus } 4 \% \text { versus } 20 \% \text { ) } \\
\text { Proportion of eyes with } \geq 2 \text {-step improvement in } \\
\text { DRSS at week } 100 \text { (62\% versus 13\%) } \\
\text { Proportion of eyes with PDR or ASNV at week } \\
100 \text { (9\% versus } 31 \%)\end{array}$ & \\
\hline VIVID/VISTA ${ }^{30-33}$ & 862 & $\begin{array}{l}\text { Cl-DMO and BCVA } \\
\text { of 24-73 ETDRS } \\
\text { letters }\end{array}$ & $\begin{array}{l}\text { IVI aflibercept } 2 \mathrm{mg} \\
2 \mathrm{q} 4 \text { versus } \\
\text { IVI aflibercept } 2 \mathrm{mg} \\
2 \mathrm{q} 8 \text { versus } \\
\text { photocoagulation }\end{array}$ & 148 weeks & $\begin{array}{l}\text { Proportion of eyes with } \geq 2 \text {-step improvement in } \\
\text { DRSS at week } 52 \text { ( } 31 \% \text { versus 12\%) } \\
\text { Proportion of eyes with } \geq 2 \text {-step improvement in } \\
\text { DRSS at week } 100 \text { (35\% versus } 13 \%) \\
\text { Proportion of eyes with PDR development at } \\
\text { week } 52 \text { ( } 2 \% \text { versus } 7 \%) \\
\text { Proportion of eyes with PDR development at } \\
\text { week } 100 \text { ( } 2 \% \text { versus 9\%) }\end{array}$ & $\begin{array}{l}\text { Analysis done with } \\
\text { pooled aflibercept group } \\
\text { versus photocoagulation }\end{array}$ \\
\hline CLARITY ${ }^{34}$ & 221 & $\begin{array}{l}\text { PDR (untreated } \\
\text { or unsuccessfully } \\
\text { treated with PRP) }\end{array}$ & $\begin{array}{l}\text { IVI aflibercept } \\
2 \text { mg versus PRP }\end{array}$ & 52 weeks & $\begin{array}{l}\text { Adjusted mean difference in BCVA at week } 52 \\
\text { (ITT: } 3.9 \text { letters [95\% Cl, 2.3-5.6]; PP: } 4.0 \text { letters } \\
\text { [95\% Cl, 2.4-5.7]) } \\
\text { Proportion of patients with no DMO at week } 52 \\
\text { (89\% versus 71\%) } \\
\text { Proportion of eyes with VH (9\% versus 18\%) } \\
\text { Proportion of eyes remained at PDR stage (78\% } \\
\text { versus } 90 \% \text { ) } \\
\text { Adjusted mean difference in QoL questionnaire } \\
\text { scores (3.0 [95\% Cl, 0.4-5.5]) }\end{array}$ & \\
\hline PROTOCOL V 35 & 702 & $\begin{array}{l}\text { Cl-DMO and BCVA } \\
\text { of at least } 79 \text { ETDRS } \\
\text { letters }\end{array}$ & $\begin{array}{l}\text { IVI aflibercept } \\
2 \text { mg versus } \\
\text { photocoagulation } \\
\text { versus observation }\end{array}$ & 2 years & $\begin{array}{l}\text { Proportion of eyes with at least a } 5 \text {-letter } \\
\text { decrease in VA at year } 2 \text { (16\% versus } 17 \% \text { versus } \\
19 \% \text { ) } \\
\text { Proportion of eyes with } \geq 2 \text {-step improvement in } \\
\text { DRSS at year } 2 \text { (14\% versus } 12 \% \text { versus 10\%) } \\
\text { Proportion of eyes with } \geq 2 \text {-step worsening in } \\
\text { DRSS at year } 2 \text { (4\% versus } 10 \% \text { versus } 11 \% \text { ) } \\
\text { Cumulative proportion of eyes with a } \\
\geq 2 \text {-worsening in DRSS over } 2 \text { years (4\% versus } \\
11 \% \text { versus } 11 \%)\end{array}$ & \\
\hline PROTOCOL AB ${ }^{36}$ & 205 & PDR and $\mathrm{VH}$ & $\begin{array}{l}\text { IVI aflibercept } 2 \text { mg } \\
\text { versus vitrectomy } \\
\text { plus PRP }\end{array}$ & 2 years & $\begin{array}{l}\text { Mean VA letter score at week } 24 \text { (59.3 [95\% Cl, } \\
54.9-63.7] \text { versus } 63.0 \text { [95\% Cl, 58.6-67.3]) } \\
\text { Mean VA letter score at week } 4 \text { (52.6 [95\% Cl, } \\
\text { 46.7-58.6] versus } 62.3 \text { [95\% Cl, 57.0-67.7]) } \\
\text { Proportion of eyes with recurrent VH at week } 24 \\
\text { (49\% versus 15\%) } \\
\text { Proportion of eyes with neovascularization on } \\
\text { clinical exam at week } 24 \text { (29.4\% versus 3.3\%) } \\
\text { Proportion of eyes with neovascularization on } \\
\text { clinical exam at year } 2 \text { (22.7\% versus 2.4\%) } \\
\text { Proportion of eyes with DMO at week } 24 \text { (8\% } \\
\text { versus 31\%) }\end{array}$ & \\
\hline
\end{tabular}

ASNV = anterior segment neovascularization; $B C V A=$ best corrected visual acuity; $C l=$ confidence interval; $C l$-DMO = centre-involved diabetic macular oedema; $D M O=$ diabetic macular oedema; DRSS = diabetic retinopathy severity scale; ETDRS = Early Treatment Diabetic Retinopathy Study; ITT = intention to treat; $I V I=$ intravitreal injection;

$N P D R=$ nonproliferative diabetic retinopathy; $P D R=$ proliferative diabetic retinopathy; $P P=$ per protocol; $P R P=$ panretinal photocoagulation; $Q 0 L=$ quality of life; $V A=$ visual acuity; $\mathrm{VH}=$ vitreous haemorrhage.

haemorrhage was higher in the PRP group than in the aflibercept group (18\% versus $9 \%, \mathrm{p}=0.034)$. A significantly higher proportion of patients in the PRP group remained at PDR (DRSS level 61 or above) compared with the aflibercept group at both 12 weeks and 52 weeks (92 [90\%] versus 81 [78\%], $p=0.016$ ). DR treatment satisfaction questionnaire scores were superior in the aflibercept group relative to the PRP group (adjusted mean difference of 3.0 [0.4-5.5, $p=0.022]$ ).

\section{The Protocol V study}

Protocol V is a multicentre, randomized, single-blind, phase III clinical trial examining long-term visual acuity outcomes in patients with $\mathrm{Cl}$ DMO and good vision (20/25 or better Snellen equivalent or at least 79 ETDRS letters). ${ }^{35}$ Improvement and worsening of DR were pre-planned exploratory outcomes. A total of 702 patients with CI-DMO and DRSS 10-75 were randomly assigned to receive treatment with intravitreal injections 
of $2 \mathrm{mg}$ of aflibercept, focal/grid photocoagulation, or be observed for 2 years. Eyes in the aflibercept group received an injection at baseline and then up to every 4 weeks as needed, as long as visual acuity or central subfoveal thickness was improved or worsened. If injections were deferred (i.e. if eye met sustained stability criteria) at three consecutive visits after 24 weeks, follow-up interval was extended to 8 and then 16 weeks. Eyes in the photocoagulation group received laser treatment at baseline, with re-treatment at 13-week intervals if indicated. Eyes in the observation group did not receive any treatment initially. If there was a visual acuity decrease in the photocoagulation or observation groups, these eyes would receive treatment with aflibercept, and re-treatment would follow the same regimen as the aflibercept group.

At year 2, the proportion of eyes with at least a 5-letter decrease in visual acuity was $16 \%, 17 \%$ and $19 \%$ in the aflibercept, photocoagulation and observation groups, respectively. No significant risk difference was found between groups. At year 2 , the proportion of eyes with a $\geq 2$-step improvement in DRSS was $14 \%, 12 \%$ and $10 \%$, respectively. No significant difference was found in relative risk (RR) between groups in adjusted treatment group comparisons (aflibercept versus photocoagulation RR, 0.90 [95\% confidence interval $\{C l\}, 0.52-1.54 ; p=0.69]$; aflibercept versus observation RR, 1.16 [95\% Cl, 0.65-2.07; $p=0.69]$; photocoagulation versus observation RR, 1.29 [95\% Cl, 0.75-2.24; $\mathrm{p}=0.69]$ ]). The proportion of eyes with a $\geq 2$-step worsening in DRSS was $4 \%, 10 \%$ and $11 \%$, respectively. No significant difference was found in RR between groups (aflibercept versus photocoagulation $\mathrm{RR}, 0.39$ [95\% $\mathrm{Cl}, 0.15-1.04 ; \mathrm{p}=0.06$ ]; aflibercept versus observation $\mathrm{RR}, 0.36$ [95\% $\mathrm{Cl}, 0.13-0.99 ; \mathrm{p}=0.05]$; photocoagulation versus observation RR, 0.90 [95\% $\mathrm{Cl}, 0.49-1.65 ; \mathrm{p}=0.74]$ ). However, over 2 years, the cumulative proportion of eyes with a $\geq 2$-step worsening in DRSS at any time was $4 \%, 11 \%$ and $11 \%$, respectively. Aflibercept was associated with a significant lower hazard ratio (HR) compared with photocoagulation and observation (aflibercept versus photocoagulation $\mathrm{HR}, 0.32$ [95\% Cl, 0.13-0.80; $\mathrm{p=0.01]}$; aflibercept versus observation $\mathrm{HR}$, $0.29[95 \% \mathrm{Cl}, 0.11-0.77 ; \mathrm{p}=0.007])$.

\section{The Protocol AB study}

Protocol $A B$ was a randomized, single-blind, clinical trial examining the effect of aflibercept versus vitrectomy with PRP on visual acuity in patients with vitreous haemorrhage from PDR. ${ }^{36}$ Two hundred and five patients with vitreous haemorrhage from PDR were randomly assigned to receive either aflibercept or vitrectomy with PRP. Eyes in the aflibercept group received intravitreal injections (2 $\mathrm{mg}$ ) at baseline and weeks 4, 8 and 12. At week 16, injection was deferred if the fundus could be viewed and neovascularization was absent. At week 24, injections were given unless the eye was stabilized. For eyes in the PRP group, vitrectomy was performed with $23 \mathrm{G}$ or smaller instruments and PRP was performed intraoperatively. Four weeks after vitrectomy, recurrent vitreous haemorrhage was treated with two monthly aflibercept injections and additional injections every 4 weeks at investigators' discretion.

The primary outcome of mean visual acuity letter score over 24 weeks was $59.3(95 \% \mathrm{Cl}$, 54.9-63.7) in the aflibercept group versus 63.0 (95\% $\mathrm{Cl}, 58.6-67.3)$ in the vitrectomy group (adjusted difference, $-5.0[95 \% \mathrm{Cl}$, -10.2 to 0.3$], \mathrm{p}=0.06$ ). The authors theorize that given the wide $\mathrm{Cl}$, the study may have been underpowered to detect a clinically important benefit in favour of initial vitrectomy with PRP.

The secondary outcome of mean visual acuity letter score at 4 weeks was $52.6(95 \% \mathrm{Cl}, 46.7-58.6)$ in the aflibercept group and $62.3(95 \% \mathrm{Cl}$, 57.0-67.7) in the vitrectomy group (adjusted difference, $-11.2[95 \% \mathrm{Cl}$, -18.5 to -3.9$], p=0.003)$. Recurrent vitreous haemorrhage occurred at least once in 48 of 97 participants (49\%) in the aflibercept group and 16 of 104 participants (15\%) in the vitrectomy group (adjusted difference, $34 \%[95 \% \mathrm{Cl}, 22-46 \%], p<0.001)$. The proportion of eyes with retinal neovascularization on clinical examination was significantly greater among participants in the aflibercept group versus participants in the vitrectomy group at 24 weeks (adjusted difference, 25\% [95\% Cl, 15$36 \%], p<0.001$ ) and at 2 years (adjusted difference, 20\% [95\% Cl, 11-30\%], $\mathrm{p}<0.001$ ). The proportion of patients in the aflibercept group with $\mathrm{Cl}-$ DMO at 24 weeks was $8 \%$ compared with $31 \%$ in the vitrectomy group (difference, -23\% [95\% Cl, -34 to -12\%], $p<0.001$ ).

The authors mention some notable limitations of this study. The protocol allowed crossover treatment for prespecified criteria, so nearly one-third of eyes in each group received both treatments. They also highlight the potential for type I error within their statistical analysis and so "findings for secondary endpoints should be interpreted as exploratory" ${ }^{36}$

\section{Impact on clinical practice}

From the evidence presented in these trials, aflibercept can have therapeutic benefit in both eyes with NPDR and eyes with PDR. Namely, preserved visual acuity, prevention of disease progression and neovascularization, regression of existing PDR to NPDR and treatment/ prevention of DR complications. With regard to visual acuity, CLARITY data suggested that in eyes with PDR, aflibercept was superior and noninferior to PRP in visual acuity outcomes as measured by BCVA. ${ }^{34}$ The VIVID/VISTA trials showed that regardless of baseline DRSS, eyes with $\mathrm{Cl}$-DMO treated with intravitreal aflibercept had significantly higher changes from baseline BCVA values relative to the photocoagulation group. The data from VIVID/VISTA also described a possible increase in treatment effect in high-risk eyes with NPDR (DRSS $\geq 53$ ) that merits further exploration. ${ }^{32,33}$

Prevention of neovascularization development and DR progression are clinically important measures of treatment efficacy. PANORAMA data indicate that aflibercept can prevent neovascularization and limit progression from NPDR to PDR relative to sham injection. ${ }^{28,29}$ Similarly, data from VIVID/VISTA show that aflibercept was associated with a significant improvement in DRSS relative to PRP in eyes with CI-DMO secondary to DR. Protocol V data suggest that aflibercept can prevent DR progression in eyes with good vision compared with photocoagulation treatment and observation..$^{35}$ In eyes with vitreous haemorrhage secondary to PDR, Protocol AB data suggested that aflibercept was not superior to vitrectomy with PRP in preventing neovascularization. ${ }^{36}$ Another consideration is the inverse: regression of neovascularization. CLARITY showed that, in eyes with PDR, aflibercept was superior to PRP in promoting regression of PDR and preventing neovascularization.

These trials also assessed the efficacy of aflibercept in preventing complications of DR, such as DMO and vitreous haemorrhage. In eyes with PDR, data from CLARITY indicated that aflibercept use was associated with less occurrence of vitreous haemorrhage and DMO relative to PRP. Similarly, in eyes with NPDR, data from PANORAMA suggest that aflibercept use was associated with less occurrence of DMO relative to sham injection. The VIVID/VISTA trials mostly consisted of eyes with NPDR, with $5 \%$ of study eyes showing signs of PDR. They arrived at a similar conclusion that aflibercept use was associated with less occurrence of vitreous haemorrhage and DMO relative to PRP.

Results from VIVID/VISTA trials informed us that anti-VEGF treatments like aflibercept are superior to photocoagulation for treatment of $\mathrm{CI}-\mathrm{DMO} .^{30}$ However, on treating vitreous haemorrhage secondary to PDR, Protocol 
$A B$ showed that there was no statistical difference in visual acuity outcomes between aflibercept versus vitrectomy with PRP. Protocol AB results suggest that aflibercept use was associated with less occurrence of DMO relative to vitrectomy with PRP. Notably, these results also dictate that aflibercept use was associated with higher recurrence of vitreous haemorrhage relative to vitrectomy with PRP. Despite the promise of aflibercept, vitrectomy with PRP resulted in a faster restoration in vision (at 4 weeks), prevention of recurrent vitreous haemorrhage and greater resolution of retinal neovascularization.

The variance in dosing regimen across each trial may help inform practice patterns. PANORAMA and CLARITY support the idea that an infrequent dosing regimen following a loading dose period may be helpful in maintaining improvement from baseline while reducing the number of injections and follow-up visits. In the CLARITY trial, during the 40 weeks of post-loading dose phase with PRN injections, there was a median of just one injection. Despite a decreased dosing regimen in the second year of the PANORAMA trial, $92 \%$ of eyes maintained their $\geq 2$-step improvement in DRSS from the first year. Based on these results and those from the VIVID/VISTA trials, Regeneron Pharmaceuticals recommends a dosage regimen of $2 \mathrm{mg} / 0.05 \mathrm{~mL}$ every 4 weeks for the first five injections and then once every 8 weeks.

The trials reviewed here suggest that aflibercept should be considered for use in both eyes with NPDR and eyes with PDR for its potential to limit DR progression and promote regression of neovascularization, as well as its association with improved visual acuity outcomes relative to PRP. The decision to use aflibercept as a treatment option versus PRP should be informed by several factors including likelihood of patient adherence in attending follow-up visits, medical comorbidities, patient age, stage of DR and probability of progression, cost of treatment and efficacy of past PRP treatments in preventing recurrent neovascularization. $\square$
1. Centers for Disease Control and Prevention. National Diabetes Statistics Report, 2020. 2020. Available at: www.cdc.gov/ diabetes/data/statistics-report/index.html (accessed 14 June 2021).

2. Zhang $X$, Saaddine JB, Chou C-F, et al. Prevalence of diabetic retinopathy in the United States, 2005-2008. JAMA. 2010:304:649-56.

3. Klein R, Klein BEK. Vision Disorders in Diabetes. In: Nationa Diabetes Data Group. Diabetes in America. National Institutes of Health. 1995:293-338.

4. Klein BEK. Overview of epidemiologic studies of diabetic retinopathy. Ophthalmic Epidemiol. 2007;14:179-83.

5. Klein R, Klein BE, Moss SE, et al. The Wisconsin epidemiologic study of diabetic retinopathy. II. Prevalence and risk of diabetic retinopathy when age at diagnosis is less than 30 years. Arch Ophthalmol. 1984;102:520-6.

6. Sabanayagam C, Banu R, Chee ML, et al. Incidence and progression of diabetic retinopathy: a systematic review. Lance Diabetes Endocrinol. 2019:7:140-9.

7. Al Ghamdi AH. Clinical predictors of diabetic retinopathy progression; a systematic review. Curr Diabetes Rev. 2020;16:242-7.

8. Willis JR, Doan Q V, Gleeson M, et al. Vision-related functiona burden of diabetic retinopathy across severity levels in the United States. JAMA Ophthalmol. 2017:135:926-32.

9. Thomas RL, Halim S, Gurudas S, et al. IDF Diabetes Atlas: a review of studies utilising retinal photography on the global prevalence of diabetes related retinopathy between 2015 and 2018. Diabetes Res Clin Pract. 2019;157:107840.

10. Yau JWY, Rogers SL, Kawasaki R, et al. Global prevalence and major risk factors of diabetic retinopathy. Diabetes Care. 2012;35:556-64

11. The Diabetic Retinopathy Study Research Group. Photocoagulation treatment of proliferative diabetic retinopathy Clinical application of Diabetic Retinopathy Study (DRS) findings, DRS Report Number 8. Ophthalmology. 1981:88:583-600.

12. Fogli S, Del Re M, Rofi E, et al. Clinical pharmacology of intravitreal anti-VEGF drugs. Eye. 2018:32:1010-20.

13. Senger D, Galli S, Dvorak A, et al. Tumor cells secrete a vascular permeability factor that promotes accumulation of ascites fluid. Science. 1983:219:983-5.

14. Witmer AN, Vrensen GFJM, Van Noorden CJF, Schlingemann RO Vascular endothelial growth factors and angiogenesis in eye disease. Prog Retin Eye Res. 2003;22:1-29.
15. Aiello LP, Avery RL, Arrigg PG, et al. Vascular endothelial growth factor in ocular fluid of patients with diabetic retinopathy and other retinal disorders. N Eng/ J Med. 1994;331:1480-7.

16. Flynn HW, Chew EY, Simons BD, et al. Pars plana vitrectomy in the Early Treatment Diabetic Retinopathy Study: ETDRS report the Early Treatment Diabetic Retinopathy Study:

77. Preti RC Ramirez LMV Monteiro MLR, at Contrast sensitivity

Preti RC, Ramirez LMV, Monteiro MLR, et al. Contrast sensitivity evaluation in high risk proliferative diabetic retinopathy treat
with panretinal photocoagulation associated or not with intravitreal bevacizumab injections: a randomised clinical trial. intravitreal bevacizumab injection:
Br J Ophthalmol. 2013:97:885-9.

18. Shimura M, Yasuda K, Nakazawa T, et al. Quantifying alterations of macular thickness before and after panretinal photocoagulation in patients with severe diabetic retinopathy and good vision. Ophthalmology. 2003;110:2386-94.

19. Michaelides M, Kaines A, Hamilton RD, et al. A prospective randomized trial of intravitreal bevacizumab or laser therapy in the management of diabetic macular edema (BOLT study) 12-month data: report 2. Ophthalmology. 2010;117:1078-86.e2

20. Nepomuceno AB, Takaki E, Paes de Almeida FP, et al. A prospective randomized trial of intravitreal bevacizumab versus ranibizumab for the management of diabetic macular edema. Am J Ophthalmol. 2013;156:502-10.e2.

21. Mitchell P, Bandello F, Schmidt-Erfurth U, et al. The RESTORE study: ranibizumab monotherapy or combined with laser versus laser monotherapy for diabetic macular edema. Ophthalmology. 2011;118:615-25.

22. Mirshahi A, Roohipoor R, Lashay A, et al. Bevacizumabaugmented retinal laser photocoagulation in proliferative diabetic retinopathy: a randomized double-masked clinical trial. Eur J Ophthalmol. 2008;18:263-9.

23. Lin J, Chang JS, Smiddy WE. Cost evaluation of panretinal photocoagulation versus intravitreal ranibizumab for proliferative diabetic retinopathy. Ophthalmology. 2016;123:1912-8.

24. Holash J, Davis S, Papadopoulos N, et al. VEGF-Trap: a VEGF blocker with potent antitumor effects. Proc Natl Acad Sci U SA 2002:99:11393-8.

25. Weidle UH, Schneider B, Georges G, Brinkmann U. Genetically engineered fusion proteins for treatment of cancer. Cancer Genomics Proteomics. 2012:9:357-72.

26. Nguyen QD, De Falco S, Behar-Cohen F, et al. Placental growth factor and its potential role in diabetic retinopathy and other ocular neovascular diseases. Acta Ophthalmol. 2018;96:e1-9.

27. Van Bergen T, Etienne I, Cunningham F, et al. The role of placental growth factor (PIGF) and its receptor system in retina vascular diseases. Prog Retin Eye Res. 2019;69:116-36.

28. ClinicalTrials.gov. Study of the Efficacy and Safety of Intravitreal (IVT) Aflibercept for the Improvement of Moderately Severe to Severe Nonproliferative Diabetic Retinopathy (NPDR) (PANORAMA). 2020. ClinicalTrials.gov Identifier: NCT02718326. (PANORAMA). 2020. ClinicalTrials.gov Identifier: NCT027 Available at: https://Clinicaltrials.gov/ct2/sh

29. Regeneron Pharmaceuticals. Diabetic Retinopathy: Anatomy Outcomes. EYLEA Improved DR Severity Scores in Patients With Moderately Severe to Severe Nonproliferative DR (NPDR). Available at: https://hcp.eylea.us/about/diabetic-retinopathyanatomy-outcomes (accessed 15 June 2021.

30. Korobelnik JF, Do DV, Schmidt-Erfurth U, et al. Intravitrea aflibercept for diabetic macular edema. Ophthalmology. 2014;121:2247-54.

31. Brown DM, Schmidt-Erfurth U, Do DV, et al. Intravitreal aflibercept for diabetic macular edema: 100-week results from the VISTA and VIVID studies. Ophthalmology. 2015;122:2044-52.

32. Mitchell P, MCAllister I, Larsen M, et al. Evaluating the impact of intravitreal aflibercept on diabetic retinopathy progression in the VIVID-DME and VISTA-DME studies. Ophthalmol Retin. 2018:2:988-96.

33. Staurenghi G, Feltgen $\mathrm{N}$, Arnold JJ, et al. Impact of baseline Diabetic Retinopathy Severity Scale scores on visual outcomes in the VIVID-DME and VISTA-DME studies. Br J Ophthalmo 2018;102:954-8

34. Sivaprasad S, Prevost AT, Vasconcelos JC, et al. Clinical efficacy of intravitreal aflibercept versus panretinal photocoagulation for best corrected visual acuity in patients with proliferative diabetic retinopathy at 52 weeks (CLARITY): a multicentre, single-blinded, randomised, controlled, phase $2 \mathrm{~b}$, noninferiority trial. Lancet. 2017;389:2193-203

35. Baker CW, Glassman AR, Beaulieu WT, et al. Effect of initial management with aflibercept vs laser photocoagulation vs observation on vision loss among patients with dianetic vs observation on vision loss among patients with diabetic macular edema involving the center of the macula and good 94.

36. Antoszyk AN, Glassman AR, Beaulieu WT, et al. Effect of intravitreous aflibercept vs vitrectomy with panretinal photocoagulation on visual acuity in patients with vitreous hemorrhage from proliferative diabetic retinopathy: randomized clinical trial. JAMA. 2020;324:2383-95. 